used to undertake nutritional assessment and measure clinical outcomes, such as prognosis. To date, no studies have reviewed the use of bioelectrical impedance analysis in head and neck cancer. This study aims to synthesise the different applications of bioelectrical impedance analysis in the clinical assessment of adults affected with advanced head and neck cancer.

Methods A systematic review of literature using five electronic databases (PubMed, Medline, EMBASE, CINAHL and PsychINFO). Search terms were based on 'head and neck cancer' and various terms relating to 'bioelectrical impedance analysis'. Empirical studies reporting the use of the different forms of bioelectrical impedance analysis in advanced head and neck cancer were included.

Results The database search identified 31 articles, of which 17 were included for full review. Bioelectical impedance analysis has been used for several purposes with patients with head and neck cancer, such as prognostication and assessments of hydration, nutrition, mucositis and fatigue. Only three articles focused specifically on the palliative phase of illness, and just one article focused on the clinical application in palliative care (predicting prognosis).

Conclusion Bioelectrical impedance analysis shows promise as a tool to assess body composition and help direct clinical care in head and neck cancer. Little evidence exists, however, for its use within palliative care and this represents an area for future focus to help with clinical decision-making and wider clinical application.

\section{EVALUATING AN 8 WEEK MINDFULNESS PROGRAMME DEVELOPED TO SUPPORT HOSPICE STAFF AND VOLUNTEERS AT LOROS HOSPICE}

Barbara Powell, Angela Baxter. LOROS Hospice

\subsection{6/spcare-2020-PCC.194}

Background Literature recognises the benefits of mindfulness practice on resilience, self-care and emotional intelligence. Front line hospice staff are at risk of compassion fatigue and burnout. An opportunity allowed us to assess the impact of an 8 week course on the well-being and self-compassion of hospice staff and volunteers.

Method A mindfulness based cognitive therapy (MBCT) course was developed encouraging participants to re-evaluate their responses to challenging situations and promoted self-care and compassion. Courses were open to all interested hospice staff and volunteers. Six courses were delivered over a ten month period. Participants completed pre and post-course WarwickEdinburgh Mental Well-being Scale (WEMWBS) questionnaires and provided free text reflection.

Results Courses involved 80 participants and four facilitators. Groups were 'closed' for the duration of the course; participants had a wide variety of roles within the hospice. WEMWBS maximum possible score $=70, \quad$ minimum $=14$; National average $=53$ (Health England Survey 2011)

53 completed pre and post questionnaires were matched and analysed.

Average pre-course score $=45.1$ (range 20-63), average postcourse score $=54.5$ (range 35-68)

Average change $=+9.3$.

$87 \%$ rated the course as of significant benefit.

98\% planned to maintain a mindfulness practice.
Free text feedback was considerable and included:

'I feel much calmer, supportive of others and it has stopped me from over-thinking situations that I can't change'

'Using different tool to adapt to the time you have'

'Being aware of thoughts and refocusing'

'Thank you for showing me I am important too and that only I can change things'

Conclusions Both WEMWBS scores and free text feedback suggest significant perceived benefit to participants. Feeling valued and more resourceful when faced with challenging situations are common themes. Long term benefits to participants and impact on patient care are still to be measured.

\section{SUBSTANCE MISUSE IN PALLIATIVE CARE: HOW WELL DO WE DOCUMENT KEY INFORMATION FOR EFFECTIVE MULTI-AGENCY WORKING ACROSS HEALTHCARE SETTINGS IN MERSEY?}

Penny Shepherd, Joanna Roberts, Kamilla Krueger, Sabine Auth, Elaine Mannering, Malcolm Cooper, Laura Chapman, Jenny Smith, Anthony Thompson. Woodlands Hospice, Willowbrook Hospice, Merseycare, Clatterbridge Cancer Centre, Marie Curie Liverpool, Countess of Chester Hospital, St Helens and Knowsley Teaching Hospitals

\subsection{6/spcare-2020-PCC.195}

Background Substance misuse (including alcohol) is a growing issue in the UK and can have far reaching effects on wellbeing, symptoms, compliance, social support and ability to access services. Medications with a potential for misuse are used commonly in palliative care. Patients with substance misuse should receive the same standard of care and treatment of symptoms as other patients. Providing this care across settings and reducing risks requires multi-agency working and good communication between healthcare professionals. Regional guidance relating to this exists in Mersey from 2009.

Aims To review current documentation of key information in patients known to palliative care with substance misuse in the Mersey region as part of updating the regional guidance.

Methods A retrospective multi-centre case-note audit was conducted including documentation of key information in patients with life limiting illness and known substance misuse receiving specialist palliative care in hospital, hospice and community settings.

Results 73 cases across the three settings were included (22\% community, 40\% Hospice, 37\% Hospital). $73 \%$ had documentation of whether drug and alcohol services were involved, $53 \%$ had documentation of whether a key worker was involved. For the 24 patients on opioid-substitution therapy (OST) 22\% had information about their community pharmacist documented, $74 \%$ had documentation of their named prescriber for OST and 63\% had documentation of their named prescriber for opioids. Documentation of communication with community teams following changes to opioid medications varied: GPs $61 \%$, drug and alcohol teams $12 \%$, and community pharmacists $5 \%$. The method of communication also varied.

Conclusions This audit highlighted inconsistent documentation of important information relating to substance misuse in patients with life limiting illness that could impair communication between healthcare professionals and services, potentially affecting the care and safety of these patients. These results have helped to inform the update of the regional guidelines in Mersey. 